\title{
A Pilot Study on the Proportion Binge Drinking Among Female Social Drinkers of Kalingalinga in Lusaka, Zambia
}

\author{
Danny Vumbi Likashi ${ }^{1}$, Ravi Paul ${ }^{1 *}$ and Luty Jason ${ }^{2}$ \\ ${ }^{1}$ Department of Psychiatry, Zambia \\ ${ }^{2}$ Locum Consultant, Liaison Psychiatry, Leicester \\ *Corresponding author: Ravi Paul, Department of Psychiatry, Zambia
}

Received: 㯺January 24, 2019

Published: 眥 January 29, 2019

\begin{abstract}
Objectives: Alcohol is a psychoactive substance with dependence-producing properties and whose harmful use causes a large burden disease, social and economic burden in societies. Binge drinking is one of the commonest forms of alcohol misuse and has been on an increase among many young women who find alcohol a source of pleasure and enjoyment when they have time out with friends and peers. Since binge drinking involves consumption of alcohol on an irregular basis, it may not be viewed as a hazardous form of alcohol use by many drinkers. The present study aimed at estimating the proportion of female binge drinkers in a population of female social drinkers in Kalingalinga township of Lusaka, the capital city of Zambia. We hypothesized that the proportion of binge drinking in a population of female social drinkers is significantly high.

Methods: Through snowball sampling, 100 questionnaires (i.e. Alcohol Use Disorders Identification Test-AUDIT) were successfully distributed to and collected from female social drinkers aged 20-39 between August and September 2016. A two-fold process was followed in identifying binge drinkers; screening for hazardous alcohol drinkers by identifying those that scored 8 points or above in the first place, and thereafter identifying binge drinking characteristics from the hazardous drinkers by following scores from the first three questions on the AUDIT.

Results: The results reviewed that 54 of the 100 participants had some form of hazardous alcohol use and 30 of the 54 hazardous drinkers possessed some binge drinking characteristics. The proportion of female binge drinkers in a population of female alcohol drinkers was estimated to be $0.556(56.6 \%)$ while in the general population it was estimated to be 0.094 which implies that $9.4 \%$ of women aged 20-39 of Kalingalinga in Lusaka, engage in alcohol binge drinking consuming on average 7-9 drinks on occasion almost on a weekly basis. Further, if 56\% of all female alcohol drinkers aged 20-39 seem to engage in some form of alcohol binge drinking, it means that that binge drinking is the highest form of alcohol misuse among these female drinkers.

Conclusion: The results of the present study suggest that there is more alcohol binge drinking among female social drinkers of Kalingalinga in Lusaka, with an estimated proportion of 0.556 (55.6\%) among female alcohol drinkers and $0.094(9.4 \%)$ in the general population of females aged between 20-39. The implication is that alcohol binge drinking seems to be the highest form of alcohol misuse among female drinkers in Kalingalinga.
\end{abstract}

Keywords: Alcohol abuse; AUDIT Questionnaires; Binge drinking (BD); Heavy Episodic drinking (HED); social drinkers

\section{Introduction}

\section{Relevance Alcohol Abuse}

Harmful alcohol use lead to about 3 million deaths per year in 2016 and that more than half of these deaths are indirectly and directly associated to binge drinking or heavy episodic drinking [1]. Although harmful alcohol use is a leading risk factor for death in males aged 15-59 [2], the evidence of women involvement in alcohol misuse and their vulnerability is a major public concern worldwide. Despite that $57 \%$ of the global population aged 15 years and older abstained from drinking alcohol, in 2016 harmful alcohol accounted for $5.3 \%$ of all deaths worldwide making mortality resulting from alcohol consumption higher than that caused by prominent diseases such as tuberculosis, HIV/AIDS and diabetes. 


\section{Classification of Alcohol Abuse}

Generally, alcohol abuse can be classified in many ways depending on the context in which the information is being used. In this study, five types of alcohol consumption (i.e. moderate alcohol consumption, binge drinking, harmful alcohol use, heavy alcohol use, low-risk drinking) have been defined to help readers differentiate the terms as they apply in alcohol studies.

Firstly, the Dietary Guidelines for Americans 2015-2020 define moderate alcohol consumption as a form of drinking that is up to one or less drinks per day for women and up to two or less drinks per day for women. Secondly, low -risk drinking involves drinking of alcohol not more than 3 drinks on any single day and no more than 7 drinks per week [3]. Harmful alcohol use, on the other hand, is alcohol consumption that results in consequences to physical and mental health and is characterized by two features: continued use despite the awareness of harmful medical and/or social effects, and a pattern of physically hazardous use of the substance [4]. Heavy alcohol use means binge drinking on five or more days in the past month [5].

Lastly, binge alcohol drinking can be a confusing concept and its use can mean different things in different contexts. Binge drinking is a kind of drinking that occurs when a person follows a pattern of drinking that bring blood alcohol concentration (BAC) to 0.08 -grams percent or above [4]. For typical adults, this pattern corresponds to consuming five or more drinks in males and four or more drinks in females, in about two hours. Binge drinking can also be defined as a pattern of heavy drinking that occurs in an extended period set aside for the purpose [6]. The terms "heavy episodic drinking (HED), bout drinking and "spree drinking" are also used as synonyms to binge drinking. All in all, the definition, 'consuming of large amounts of alcohol on an irregular basis' [7] seems to be practical for a developing country like Zambia where the consumption of non-quantified alcoholic beverages is commonplace.

\section{The Proportion of Alcohol Binge Drinking Globally}

The proportion of binge drinking, like many other forms of alcohol consumption, varies tremendously by region, race and sex. Generally, in Muslim regions such as North Africa and the Middle East, alcohol consumption is very low such that for some countries consumption is close to. On the other hand, countries in Eastern Europe are the highest in alcohol consumption with figures between 14-17 liters of alcohol per person per year. The proportion of binge drinking seem to be different from the general alcohol consumption. Some countries where overall alcohol consumption is low, have high percentages of binge drinking. In Madagascar, more than two thirds (65\%) of drinkers have heavy episodes of drinking in the previous month.

Intraregional differences are also evident: in Italy only $6 \%$ of drinkers had heavy episodes of drinking in contrast to nearly half in Ireland, $42 \%$ in Belgium, one third in UK and France, and 20\% in Spain [8]. The general alcohol consumption by gender shows significant differences with men consuming more alcohol than women. Although binge drinking is common among males than females, studies report that young women have begun to show drinking patterns similar to those of their male peers, especially regarding heavy episodic drinking [9].

\section{Binge Drinking in Zambia}

World Health Organization's country profile reports for Zambia, both 2014 and 2018, show that heavy episodic drinking (binge drinking) is higher in males than females [1]. The proportions of binge drinking are high in late adolescence and early adulthood though the sex ratio is different from the general population especially among student populations where an estimated $45.1 \%$ females compared to $38.7 \%$ males get drunk [6]. This is remarkable because in the general population, non-student female adolescents and young adults have a much lower tendency to binge drink compared to their male peers. About $30-50 \%$ of all women drinkers in South Africa, Zambia and Chad are said to be binge drinkers [10]. Topics on alcohol use in Zambia are highly unexplored and many issues related to alcohol have remained elusive to academia. Nevertheless, Zambia is among the nations with the highest levels of drinking in Africa [11]. Findings by the WHO published in the Washington Post, indicated that there is no nation that has harderdrinking women in the world than Zambia [12]. An issue that needs extra research to ascertain the truth.

\section{The Significance of Alcohol Binge Drinking aAmong Female Drinkers in Zambia}

Female alcohol drinkers are more likely to be affected by alcohol use disorders than male drinkers. Because of having a higher concentration of fat in body tissue, females tend to reach a higher alcohol consumption than male drinkers [13]. This in turn makes it possible for female drinkers to metabolize alcohol much faster than males [14]. It should as well be noted that the pharmacological impact of a given dose of alcohol is greater in women than females implying that female alcohol drinkers are at a higher risk of alcohol use disorders than male drinkers [15]. In 2016 the leading contributors of to the burden of alcohol-attributable deaths among men included injuries, digestive diseases and alcohol use disorders, whereas among women the main contributors were cardiovascular diseases, digestive diseases and injuries.

In a Zambian society, like many other African countries, a typical woman is a multitask taker managing households, children, jobs and extended family relationships. Because most habitual binge drinkers may have false beliefs in their behavioral tolerance to the impairing effects of alcohol [16], binge drinking females could be at risk of road and household accidents, involvement in violence, unsafe sex practices, among other things. Therefore, a study on binge drinking in female social drinkers is a viable 
endeavor because it addresses an issue that not only affects the female drinker but engulfs issues affecting family life and the wider society, which the female is part of.

\section{Methods}

\section{Sampling}

By using snowball sampling method, 100 questionnaires were successfully distributed to female social drinkers aged between 20 and 39. The sampling method was best suited to this study because there is some stigma that is attached to alcohol consumption in Zambia which makes it very difficult for most women to openly acknowledge that they consume alcohol. To identify binge drinkers, the study took a two-fold approach; identification of hazardous drinking in all the 100 participants in the first place, followed by screening for binge drinking characteristics. The sampling was preceded by dividing the study area into four and then identifying female drinkers from each of the areas who would roll out to other drinkers.

From the 100 questionnaires, 54 were screened as indicating some form of hazardous drinking because of scoring 8 points and above. The 54 questionnaires were further screened to identify characteristics of binge drinking by using questions 1, 2 and 3. 30 females were qualified as being alcohol binge drinkers based on the three indices of alcohol consumption; frequency, quantity and intensity of drinking which match questions 1, 2 and 3 of the AUDIT questionnaires. To make the study beneficial to the participants, those identified as possessing some hazardous drinking patterns were advised to seek psychosocial help from University Teaching Hospital (UTH) or from other Mental Health care providers within Lusaka. By using the phone numbers provided at the end of the questionnaires, participants were contacted and advised to seek medical advice from UTH where arrangements were already made for them.

\section{Measures}

Data was collected through hand-delivered questionnaires (the Alcohol Use Identification Tool (AUDIT) questionnaires). The AUDIT questionnaire is a well validated tool developed by the World Health Organization (WHO) to assess alcohol consumption, drinking behaviors, and alcohol related problems. The questionnaire has two versions; the clinician administered and the self-administering versions. In this study, the self-administered version was translated into the local language, Nyanja, and used side by side the ones in English. Going by the major definitions on binge drinking reviewed earlier, three indices of alcohol usage were identified and associated to three main questions on the AUDIT. These were; frequency of drinking, quantity of alcohol an individual drink and the intensity of drinking.

From the AUDIT questionnaire the frequency of drinking is determined by question 1 "How often do you have a drink containing alcohol? (Never/ Monthly or less/2 to 4 times a month/2 to 3 times a week/4 or more times a week)." Second, third and fourth responses (Monthly or less, 2 to 4 times a month, 2 to 3 times a week) are characteristic of binge drinking because of the episodes per month or week $[17,18]$. Those that drink daily or almost daily for example, were disqualified because they fall under the category of chronic drinkers.

The quantity of drinking was determined by question 2 from the questionnaire; "How many drinks containing alcohol do you have on a typical day when you are drinking? ( 1 or 2,3 or 4,5 or 6 , 7, 8, or 9, 10 or more)." Finally, the intensity of alcohol drinking was determined by question 3. "How often do you have 6 or more drinks on a single occasion? (Never /Less than monthly /Weekly / Daily or almost daily).

\section{Results}

\section{Demographic Data of Participants}

The main characteristics considered were age, years of education, presence/absence of impairments and alcohol drinking. The mean age for the study participants was 27 with a standard deviation of 5.456 . On average $43 \%$ of the participants had completed secondary education (Table 1).

Table 1: Demographic Data for Participants.

\begin{tabular}{|c|c|c|}
\hline Variable & $\begin{array}{l}\text { Number of } \\
\text { Participants }\end{array}$ & Percentage \\
\hline \multicolumn{3}{|l|}{ Age $(N=30$, Mean $=27.00, S D=5.456)$} \\
\hline a. $20-24$ & 12 & $40 \%$ \\
\hline b. $25-29$ & 8 & $27 \%$ \\
\hline c. $30-34$ & 6 & $20 \%$ \\
\hline \multirow[t]{2}{*}{ d. $\geq 35$} & 4 & $13 \%$ \\
\hline & & $100 \%$ \\
\hline $\begin{array}{l}\text { Education Level } \\
\text { a. Primary ( } 1 \leq 7 \text { years of schooling) }\end{array}$ & 10 & $33.30 \%$ \\
\hline $\begin{array}{l}\text { b. Secondary ( } 8 \leq 12 \text { years of } \\
\text { schooling) }\end{array}$ & 13 & $43.30 \%$ \\
\hline \multirow[t]{2}{*}{ c. Tertiary ( $\geq 12$ years of schooling) } & 7 & $23.30 \%$ \\
\hline & & $100 \%$ \\
\hline $\begin{array}{l}\text { Alcohol Drinking } \\
\text { a. Days/Week }\end{array}$ & 1 day & \\
\hline b. Number of drinks/Occasion & 6 drinks & \\
\hline c. $\geq$ Four drinks/Occasion & Weekly & \\
\hline
\end{tabular}

\section{Characteristics of Binge Drinking}

In order to identify who engaged in alcohol binge drinking, the study identified three characteristics of binge drinking which cut across many scientific definitions of this form of drinking which were reviewed in other scholars' works. The characteristics 
were frequency of drinking, quantity of drinking and intensity of drinking (Table 2).

Table 2: Characteristics of Binge Drinking among participants.

\begin{tabular}{|c|c|c|}
\hline characteristic & Mean & Standard Deviation \\
\hline Frequency & 2.17 & 0.834 \\
\hline Quantity & 3.03 & 0.964 \\
\hline Intensity & 2.53 & 0.507 \\
\hline
\end{tabular}

The scores for these variables were: frequency $(M=2.17$, $\mathrm{SD}=.834)$, quantity $(\mathrm{M}=3.03, \mathrm{SD}=.964)$ and intensity $(\mathrm{M}=2.53$, $\mathrm{SD}=.507)$. The mean of 2.17 represents response (2) on the questionnaire implying that an average female took an alcoholic drink ' 2 to 4 times per month.' This does not represent regular drinking but irregular type of drinking characteristic of binge drinking. Further, quantity is represented by a mean score of 3.03 translating into ' 7,8 or 9 drinks' per occasion, implying consumption of large amounts of alcohol on one sitting. Besides, the intensity of drinking was represented by an average of 2.53 which meant that taking of six or more alcoholic drinks was almost on a weekly basis.

\section{Proportion of Binge Drinkers in a Population of Female Alcohol Drinkers}

The proportion of binge drinkers from the sampled number of social drinkers was calculated by using the formula:

$$
P=x / n
$$

Where,

$\mathrm{p}=$ population proportion

$\mathrm{x}=$ the number of items you are interested in (i.e. female binge drinkers)

$\mathrm{n}=$ the total number of in the population (i.e. all female social drinkers)

$$
\mathrm{p}=30 / 54=0.556
$$

\section{Proportion of Binge Drinking in the General Population}

The pie-chart below helps to show the proportion of alcohol binge drinking in a total of 54 female social drinkers whose AUDIT questionnaires were well completed and collected (Figure 1). From a total of 54 social drinkers, 30 were classified as binge drinkers accounting for $56 \%$ of all female drinkers aged 20-39. About 24 did not meet the criteria of being classified as binge drinkers based on the three indices of alcohol consumption; frequency, quantity and intensity of drinking, determined using the AUDIT questionnaire. The 24 mainly comprised of chronic drinkers who consumed alcohol almost on a daily basis and were referred to the University Teaching Hospital for psychosocial help. Psychotherapy, both group and individual, is recommended for the treatment of alcohol dependence (addiction) which was observed in the 24 participants
[4]. It involves educating patients on the risks of continued alcohol use and requesting the patient to resume personal responsibility for change.

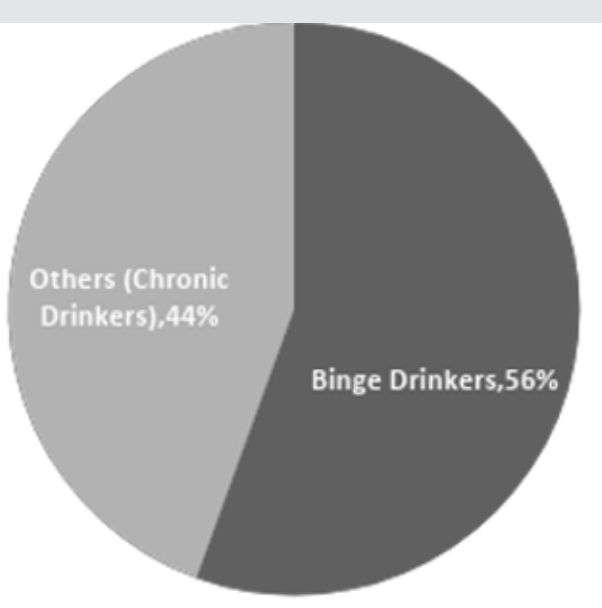

Binge Drinkers Others (Chronic Drinkers)

Figure 1: Proportion of Female Binge Drinking.

The proportion of binge drinkers in the general population of female social drinkers was estimated by using the formula below.

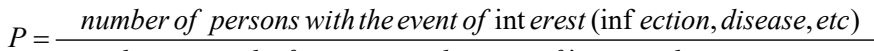
population at risk of presenting the event of int erest during a given time

$\mathrm{P}=$ number of potential BDs number of females aged between 20-39

$\mathrm{P}=7107553$

$\mathrm{P}=0.094$

\section{Discussion}

The results match other studies which gave the same definition of binge drinking. The definitional characteristic of binge drinking of 'consuming large amounts of alcohol on irregular basis' 9 that was described earlier is such an example. The clinical definition of binge drinking as the 'drinking of alcohol that brings blood alcohol concentration (BAC) to 0.08-gram percent or above [3], is another key example that match the results of this study because the definition indicates that blood alcohol level of 0.08-gram percent or above is only reached by taking 5 or more drinks or more drinks (in males) or 4 or more drinks (in females) on an occasion' [16].

\section{Estimated Proportion of Binge Drinking}

The population of all female inhabitants in Kalingalinga is around 20194 according to 2010 Census [19] and those aged between 20-39 are only 7553 females. Further, about $16.8 \%$ of the female population comprise alcohol drinkers1 translating into 1268 potential female drinkers. Going by the results in figure $1,56 \%$ of 1268 female drinkers is about 710 females who are potential binge drinkers. Therefore, the proportion of binge 
drinking in Kalingalinga can be estimated to be 0.094. Expressed as a percentage, the proportion of alcohol binge drinking is about $9.4 \%$ of all females aged between $20-39$.

The results of the present study have a few similarities and contradictions with some other studies that were reviewed. In 2011, more than 13.6 million (12.5\%) U.S. adult women binge drank an average of three times a month (frequency) and consumed on average six drinks on occasion (intensity). The prevalence and intensity of binge drinking was highest among women aged 18-24 years. Africa has the world's highest proportion of binge drinkers with about $25 \%$ of its population engaging in alcohol binge drinking despite its large numbers of Muslims and evangelical Christians who abstain from alcohol [20]. Among women who drink in South Africa, Zambia and Chad 30-50\% report binge drinking yet the prevalence of women drinking alcohol in these countries ranges from $15-30 \%$ [10]. Binge drinking is mirrored here as being the highest form of excessive alcohol drinking in females in Africa and also Zambia [21].

In the case of Kalingalinga the results seem to suggest that about $9.4 \%$ of women aged 20-39 years engage in alcohol binge drinking consuming on average 7-9 drinks on occasion almost on a weekly basis. Further, of all female alcohol drinkers, binge drinkers comprise the highest group with about 56\% higher than the percentage projected by Culley and others in 2013. Therefore, the proportion rate of alcohol binge drinking among females of Kalingalinga is estimated to be at $9.4 \%$ in the general population for females aged between $20-39$ years and $56 \%$ among all potential female drinkers [22,23].

\section{Limitations}

In terms of limitations, the first challenge was refusal by most women above 35 years to participate in the study due to the stigma that is attached to female alcohol consumption because Zambian culture does not approve of women engagement in alcohol drinking. Secondly, defining binge drinking was another challenge as there is no standard definition for this form of alcohol consumption. However, the review of many available definitions helped to come up with three many indices of alcohol consumptions which were typical of binge drinking; frequency of drinking, quantity of consumed alcohol and the intensity of drinking.

\section{Conclusion}

Binge drinking, which involves consuming large amounts of alcohol on an irregular basis, is one of the commonest forms of alcohol misuse among adolescent girls and young women. The study investigated the proportion of binge drinking among female alcohol drinkers aged between 20-39 of Kalingalinga in Lusaka, the capital city of Zambia. In conclusion, the results of the present study suggest that there is more alcohol binge drinking among female social drinkers of Kalingalinga in Lusaka, with a proportional rate of $9.4 \%$ in the general population of females aged between 20-39 and $56 \%$ among female alcohol drinkers of this age-group. The implication is that alcohol binge drinking seems to be the highest form of alcohol misuse among female drinkers in Kalingalinga.

The study has been a viable endeavor as it has provided some estimation on the proportion of alcohol consumption among females which can be important to policy makers and the general public. The pattern of drinking reported by participants can be a source of worry because this is capable of increasing the country's disease burden in the next few years if no measures are put in place. Further, the drinking pattern puts the female drinkers at risk of road and home accidents, exposure to sexually transmitted diseases and HIV/AIDS, and that the general outcome of alcohol abuse will impinge on their multi-task taking skills. All these effects mentioned here can exacerbate poverty levels in the Zambian society simply because the whole society highly depends on the woman for its operations. There is need to carry out more research in other parts of Lusaka in order to obtain data that is generalizable and can help policy makers to act on this form of drinking that is likely to deter economic development, increase disease burden and affect family life, among others. Another viable proposition can be a 'comparative investigation in the proportion of HIV/AIDS among female alcohol drinkers and their non- drinking counterparts.'

\section{References}

1. World Health Organisation (2018) Global Status Report on Alcohol and Health.

2. Grucza RA, Bucholz KK, Rice JP, Bierut LJ (2008) "Secular Trends in the Lifetime Prevalence of Alcohol Dependence in the United States: A Reevaluation." Alcoholism: Clinical and Experimental Research 32(5): 763770 .

3. National Institute on Alcohol Abuse and Alcoholism (2013) NIAAA Newsletter, Winter 2004 Number 3. NIAAA Council Approves Definitions of Binge Drinking 2004.

4. Ahuja NA (2006) Short Textbook of Psychiatry. Jaypee Brothers, New Delhi, India.

5. The Substance Abuse and Mental Health Services AdministrationSAMHSA (2015) Binge Drinking: Terminology and Patterns of Use. SAMHSA, Rockville, Maryland.

6. World Health Organization (2014) Global Status Report on Alcohol and Health, Geneva.

7. Kokavec A, Crowe SFA (1999) Comparison of Cognitive Performance in Binge Versus Regular Chronic Alcohol Misusers. Alcohol 34(4): 601-68.

8. Hannah R, Max R (2018) Alcohol Consumption.

9. Andersson C, Sundh V, Waern M, Jakobson A, Lissner L, et al. (2013) "Drinking context and problematic alcohol consumption in young Swedish women." Addiction Research and Theory 21(6): 457-468.

10. Culley CL, Ramsey TD, Mugyenyi G, Kiwanuka GN, Ngonzi J, et al. (2013) "Alcohol Exposure Among Pregnant Women in Sub-Saharan Africa: A Systematic Review." J Popul Ther Pharmacol 20(3): 321-333.

11. Kabuba N, Menon AJ, Hestad K (2011) "Moderate Alcohol Consumption and Cognitive Functioning in a Zambian Population." Medical Journal of Zambia 38(2)

12. Mwale S (2014) "Zambian Women Lead in Alcohol Consumption." Times of Zambia Forward with the Nation. 
13. De Bellis MD, Narasimhan A, Thatcher DL, Keshavan MS, Soloff P, et al. (2005) "Prefrontal Cortex, Thalamus, a Cerebellar Volumes in Adolescent and Young Adults with Adolescent-onset Alcohol Use Disorders and Comorbid Mental Disorders." Alcoholism: Clinical and Experimental Research 29(9): 1590-1600.

14. Lisdahl KM, Thayer R, Squeglia LM, McQueeny TM, Tapert SF (2013) "Recent Binge Drinking Predicts Smaller Cerebellar Volumes in Adolescents." Psychiatry Research: Neuroimaging 211(1): 17-23.

15. Institute of Alcohol Studies (2007) Binge Drinking: Medical and Social Consequences.

16. Brumback T, Cao D, King A (2007) Effects of Alcohol on Psychomotor Performance and Perceived Impairment in Heavy Binge Social Drinkers." Drug Alcohol Depend 91(1): 10-17.

17. Korhonen M (2004) Alcohol Problems and Approaches: Theories, Evidence and Northern Practice.
18. Cranford JA, McCabe SE, Boyd CJ (2006) "A New Measure of Binge Drinking: Prevalence and Correlates in a Probability Sample of Undergraduates." Alcoholism: Clinical and Experimental Research 30(11): 1896-1905.

19. Central Statistics Office (2012) 2010 Census of Population and Housing Descriptive Tables: Lusaka Province, Zambia.

20. Motsoeneng T (2012) "Addiction." Binge Drinking in Africa a crisis Johannesburg: Reuters Health.

21. Berridge V, Thom B, Herring R (2007) The Normalization of Binge Drinking? A Historical and Cross-cultural Investigation with Implications for Action. Unpublished final report for AERC.

22. Kanny D, Liu Y, Brewer RD, Lu H (2013) Prevention Binge DrinkingUnited States.

23. World Health Organisation (2001) Alcohol Use Disorders identification Test (AUDIT), Geneva.

\section{(a)

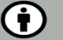 \\ This work is licensed under Creative Commons Attribution 4.0 License}

To Submit Your Article Click Here: Submit Article

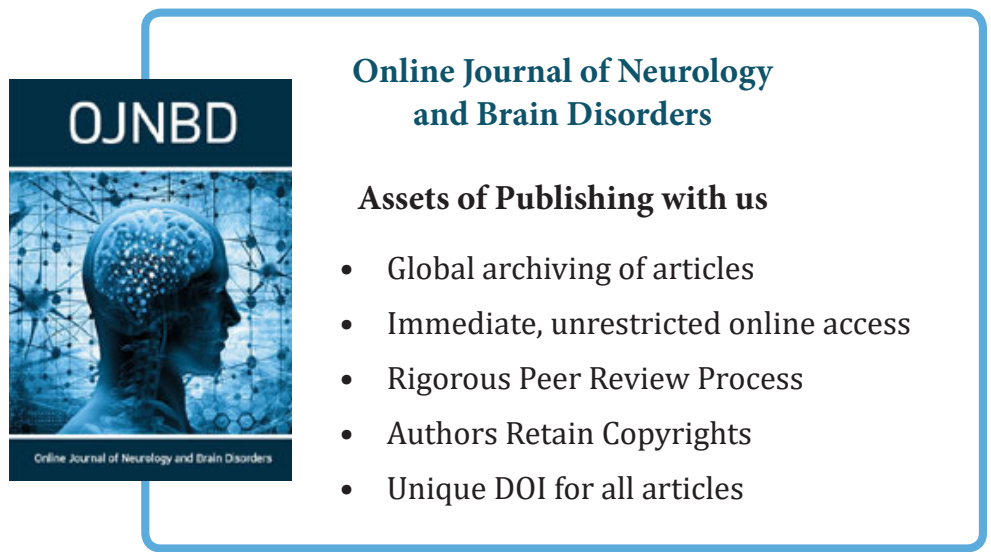

